\title{
Aerobic Anaerobic WWTP Design Simulation in the Jeans Industry to Reduce TSS Levels according to Quality Standards
}

\author{
E Suhartono $^{1}$, S Setyowati Rahayu ${ }^{2}$ B Setyobudi $^{1} \&$ M YuSa $^{3}$ \\ ${ }^{1}$ Department of Civil Engineering, State Polytechnic of Semarang \\ ${ }^{2}$ Department of Mechanical Engineering, State Polytechnic of Semarang \\ ${ }^{3}$ Department of Informatics, University of Bengkulu \\ Indonesia
}

\begin{abstract}
The rapid development of the textile industry has induced an increase in environmental pollution. This is not only harmful to the environment but is also a threat to living things, especially in humans. Dyes from textile waste when disposed into the water, pollute its surface thereby preventing sunlight into it. The level of organic compounds in water can be measured by TSS parameters. In general, the discarded TSS level ranges from about 475 to $678 \mathrm{mg} / \mathrm{L}$. According to the Provincial Regulation of Central Java Number 5 of 2012 on Amendments to the Regional Regulation of Central Java Number 10 of 2004 on Wastewater Quality Standards, the maximum allowable level for Textile and Batik Wastewater for TSS is 50mg/L. The Aerobics Anaerobic WWTP Design function is to reduce TSS levels in accordance with quality standards. The simulation results showed that, at the discharge level of $50 \mathrm{m3} / \mathrm{day}$, the initial TSS level of $678 \mathrm{mg} / \mathrm{L}$ decreases to $40.7 \mathrm{mg} / \mathrm{L}$ in accordance with the established Quality Standards and become an environmentally friendly Industry.
\end{abstract}

Kkeywords: Wastewater, Jeans Industry, Aerobic Anaerobic WWTP, Environmentally Friendly.

\section{INTRODUCTION}

"Ryan Jeans" Small and Medium Enterprises (SME) at Surabayan Wonopringgo Pekalongan still survives even on this year, the production is getting increase and increase and the marketing increases as much as $10 \%$ of the increasing sum of production/days from 45 score/day become 50 score/day, so that the profit increases $10 \%$. We did the dedication for community with scheme Regional Superior Product Development Program (PPUD) in Kabupaten Pekalongan. In 2020 by adding Appropriate technology (TTG) in the form of clean water supply, Appropriate technology (TTG) dryer, and Appropriate technology (TTG) Showroom with $4 \times 6 \mathrm{~m}^{2}$ in size and medicine room also Office and Coloring Training with natural dyes (indigo) and wearing Personal Protective Equipment (PPE) for the employees

\section{ACTIVITY METHOD}

We accompany "Ryan Jeans" Small and Medium Enterprises (SME) at Surobayan Wonopringgo Pekalongan by giving brief and discussion for getting the increase of production and marketing by using website and facebook also fixing production become green product through appropiate technology. Beside that we are also doing activity by :

a. Designing Wastewater Treatment Plant (WWTP) aerobic anaerobic system as alternative wastewater treatment plant of washing jeans before discharging into the environment.

b. Total Suspended Solid (TSS) in the textile industry commonly high that is about $678 \mathrm{mg} / \mathrm{L}$, while the aquatic environment only can receive TSS about $50 \mathrm{mg} / \mathrm{L}$ until $400 \mathrm{mg} / \mathrm{L}$. 
International Journal of Advances in Scientific Research and Engineering (ijasre), Vol 6 (8), August -2020

c. Wastewater sample with the wastewater volume $50 \mathrm{~m}^{3} /$ day and contain TSS $678 \mathrm{mg} / \mathrm{L}$.

d. TSS calculation resulting from experimental simulation.

\section{RESULTS AND DISCUSSION}

Wastewater Treatment Plant with the Aerobic Anaerobic System mostly found at hospital wastewater treatment which is more infectious than wastewater from other activities, as shown below

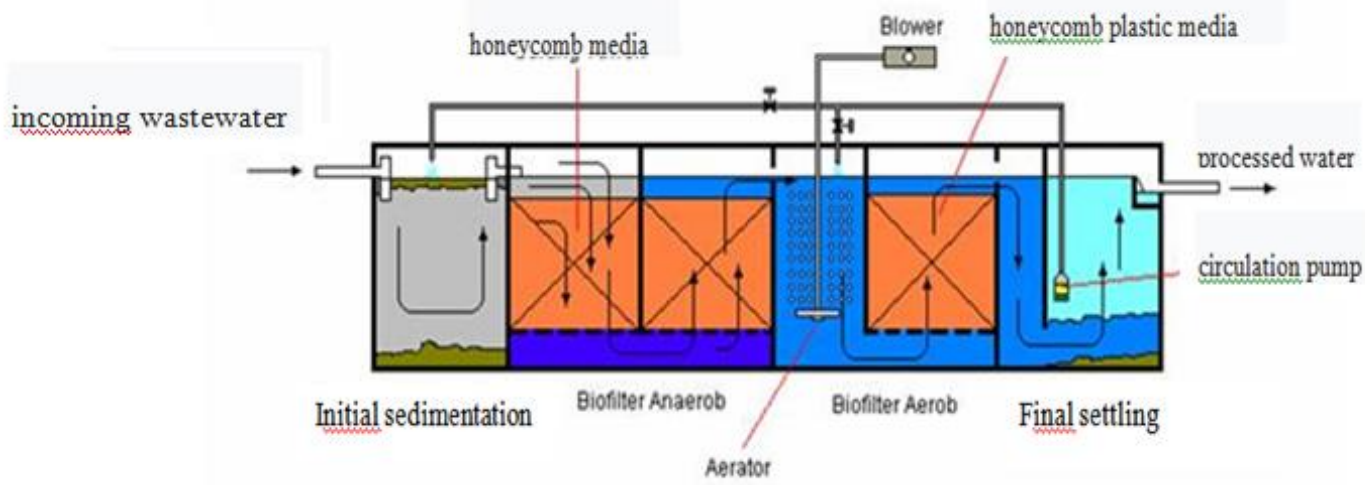

Figure 1. Anaerobic Aerobic WWTP

For discharger wastewater $50 \mathrm{~m}^{3}$ and $\mathrm{TSS}=50 \mathrm{mg} / \mathrm{L}$

Table 1. Wastewater with TSS $50 \mathrm{mg} / \mathrm{L}$

\begin{tabular}{|c|c|c|c|c|c|c|c|c|}
\hline \multirow[b]{2}{*}{ Name of Tub } & \multirow{2}{*}{$\begin{array}{l}\text { DT } \\
\text { (hour } \\
\text { ) }\end{array}$} & \multirow{2}{*}{$\begin{array}{l}\text { Efficien } \\
\text { cy } \\
\quad(\%)\end{array}$} & \multirow{2}{*}{$\begin{array}{l}\text { Deb } \\
\text { it } \\
\left(\mathrm{m}^{3} /\right. \\
\text { day })\end{array}$} & \multirow{2}{*}{$\begin{array}{c}\text { Effecti } \\
\text { ve } \\
\text { volum } \\
\text { e } \\
\left(\mathbf{m}^{\mathbf{3}}\right)\end{array}$} & \multicolumn{3}{|c|}{ Dimension } & \multirow{2}{*}{$\begin{array}{l}\text { TSS } \\
\text { results } \\
(\mathrm{mg} / \mathrm{L})\end{array}$} \\
\hline & & & & & $\begin{array}{l}\mathbf{L}( \\
\mathbf{m})\end{array}$ & $\begin{array}{l}\text { W( } \\
\text { m) }\end{array}$ & $\begin{array}{l}\text { Height } \\
\text { + Air } \\
\text { ( m) }\end{array}$ & \\
\hline Like equalization & 5.12 & 0 & 50 & 1,0 & 2,2 & 1 & 1,4 & 50 \\
\hline $\begin{array}{l}\text { Initial } \\
\text { sedimentation }\end{array}$ & 3 & 25 & 50 & 10,7 & 3,3 & 4 & 2,4 & 37.5 \\
\hline Anaerob & 9 & 80 & 50 & 6,3 & 0,7 & 4 & 2,4 & 7.5 \\
\hline Aerob & 5.6 & 60 & 50 & 18,8 & 2,0 & 4 & 2,4 & 3 \\
\hline $\begin{array}{l}\text { Final } \\
\text { Sedimentation }\end{array}$ & 3 & 0 & 50 & 6,3 & 0,7 & 4 & 6,3 & 3 \\
\hline
\end{tabular}

Table 1 shows the early TSS was $50 \mathrm{mg} / \mathrm{L}$, after receiving Aerobic Anaerobic WWTP constant in $3 \mathrm{mg} / \mathrm{L}<50 \mathrm{~m} / \mathrm{L}$ (qualify for First-Class river quality standard according to PP 82/2001) 
International Journal of Advances in Scientific Research and Engineering (ijasre), Vol 6 (8), August -2020

Table 2. Wastewater with TSS $150 \mathrm{mg} / \mathrm{L}$

\begin{tabular}{|c|c|c|c|c|c|c|c|c|}
\hline \multirow[b]{2}{*}{ Name of Tub } & \multirow{2}{*}{$\begin{array}{l}\text { DT } \\
\text { (hour } \\
\text { ) }\end{array}$} & \multirow{2}{*}{$\begin{array}{l}\text { Efficien } \\
\text { cy } \\
(\%)\end{array}$} & \multirow[b]{2}{*}{$\begin{array}{c}\text { Deb } \\
\text { it } \\
\left(\mathbf{m}^{3} /\right. \\
\text { day })\end{array}$} & \multirow{2}{*}{$\begin{array}{c}\text { Effecti } \\
\text { ve } \\
\text { volum } \\
\mathbf{e} \\
\left(\mathbf{m}^{\mathbf{3}}\right)\end{array}$} & \multicolumn{3}{|c|}{ Dimension } & \multirow[b]{2}{*}{$\begin{array}{c}\text { TSS } \\
\text { result } \\
\text { s } \\
(\mathbf{m g} / \\
\mathbf{L})\end{array}$} \\
\hline & & & & & $\begin{array}{l}\mathbf{L}( \\
\mathbf{m})\end{array}$ & $\begin{array}{l}\text { W( } \\
\text { m) }\end{array}$ & $\begin{array}{c}\text { Heig } \\
\text { ht + } \\
\text { Air } \\
\text { (m) }\end{array}$ & \\
\hline Equalization & 5.12 & 0 & 50 & 1,0 & 2,2 & 1 & 1,4 & 150 \\
\hline $\begin{array}{l}\text { Initial } \\
\text { sedimentation }\end{array}$ & 3 & 25 & 50 & 10,7 & 3,3 & 4 & 2,4 & 112.5 \\
\hline Anaerob & 9 & 80 & 50 & 6,3 & 0,7 & 4 & 2,4 & 22.5 \\
\hline Aerob & 5.6 & 60 & 50 & 18,8 & 2,0 & 4 & 2,4 & 9 \\
\hline $\begin{array}{l}\text { Final } \\
\text { Sedimentation }\end{array}$ & 3 & 0 & 50 & 6,3 & 0,7 & 4 & 6,3 & 9 \\
\hline
\end{tabular}

Table 2 shows the early TSS was $150 \mathrm{mg} / \mathrm{L}$, after the wastewater treatment Aerobic Anaerobic WWTP constantly become $9 \mathrm{mg} / \mathrm{L}<50 \mathrm{mg} / \mathrm{L}$ (qualify for First-Class river quality standard according to PP $82 / 2001)$

Table 3. Wastewater with TSS $250 \mathrm{mg} / \mathrm{L}$

\begin{tabular}{|c|c|c|c|c|c|c|c|c|}
\hline \multirow[b]{2}{*}{ Name of Tub } & \multirow{2}{*}{$\begin{array}{l}\text { DT } \\
\text { (hour } \\
\text { ) }\end{array}$} & \multirow{2}{*}{$\begin{array}{l}\text { Efficien } \\
\text { cy } \\
(\%)\end{array}$} & \multirow{2}{*}{$\begin{array}{l}\text { Debit } \\
\left(\mathrm{m}^{3} /\right. \\
\text { day })\end{array}$} & \multirow{2}{*}{$\begin{array}{c}\text { Effec } \\
\text { tive } \\
\text { volu } \\
\text { me } \\
\left(\mathrm{m}^{3}\right)\end{array}$} & \multicolumn{3}{|c|}{ Dimension } & \multirow{2}{*}{$\begin{array}{l}\text { TSS } \\
\text { results } \\
\text { (mg/L) }\end{array}$} \\
\hline & & & & & $\begin{array}{l}\mathrm{L}( \\
\mathrm{m})\end{array}$ & $\begin{array}{l}\text { W( } \\
\mathrm{m})\end{array}$ & $\begin{array}{c}\text { Heigh } \\
\mathrm{t}+ \\
\text { Air } \\
(\mathrm{m})\end{array}$ & \\
\hline Equalization & 5.12 & 0 & 50 & 1,0 & 2,2 & 1 & 1,4 & 250 \\
\hline $\begin{array}{l}\text { Initial } \\
\text { sedimentation }\end{array}$ & 3 & 25 & 50 & 10,7 & 3,3 & 4 & 2,4 & 187.5 \\
\hline Anaerob & 9 & 80 & 50 & 6,3 & 0,7 & 4 & 2,4 & 37.5 \\
\hline Aerob & 5.6 & 60 & 50 & 18,8 & 2,0 & 4 & 2,4 & 15 \\
\hline $\begin{array}{l}\text { Final } \\
\text { Sedimentation }\end{array}$ & 3 & 0 & 50 & 6,3 & 0,7 & 4 & 6,3 & 15 \\
\hline
\end{tabular}

Table 3 shows the early TSS was $250 \mathrm{mg} / \mathrm{L}$, after processed using Aerobic Anaerobic WWTP become $15 \mathrm{mg} / \mathrm{L}<50 \mathrm{mg} / \mathrm{L}$ (qualify for First-Class river quality standard according to PP 82/2001) 
Table 4. Wastewater with TSS $500 \mathrm{mg} / \mathrm{L}$

\begin{tabular}{|c|c|c|c|c|c|c|c|c|}
\hline \multirow[b]{2}{*}{ Name of Tub } & \multirow{2}{*}{$\begin{array}{c}\text { DT } \\
\text { (hour) }\end{array}$} & \multirow{2}{*}{$\begin{array}{l}\begin{array}{l}\text { Effici } \\
\text { ency }\end{array} \\
(\%)\end{array}$} & \multirow{2}{*}{$\begin{array}{l}\text { Debit } \\
\left(\mathrm{m}^{3} /\right. \\
\text { day })\end{array}$} & \multirow{2}{*}{$\begin{array}{c}\text { Effecti } \\
\text { ve } \\
\text { volum } \\
\text { e } \\
\left(\mathrm{m}^{3}\right)\end{array}$} & \multicolumn{3}{|c|}{ Dimension } & \multirow{2}{*}{$\begin{array}{l}\text { TSS } \\
\text { results } \\
\text { (mg/L) }\end{array}$} \\
\hline & & & & & $\begin{array}{l}\mathrm{L}( \\
\mathrm{m})\end{array}$ & $\begin{array}{l}\mathrm{W}( \\
\mathrm{m})\end{array}$ & $\begin{array}{c}\text { Heigh } \\
\mathrm{t}+ \\
\text { Air } \\
(\mathrm{m})\end{array}$ & \\
\hline $\begin{array}{l}\text { Like } \\
\text { equalization }\end{array}$ & 5.12 & 0 & 50 & 1,0 & $\begin{array}{l}2 \\
2\end{array}$ & 1 & 1,4 & 500 \\
\hline $\begin{array}{l}\text { Initial } \\
\text { sedimentation }\end{array}$ & 3 & 25 & 50 & 10,7 & $\begin{array}{l}3, \\
3\end{array}$ & 4 & 2,4 & 375.0 \\
\hline Anaerob & 9 & 80 & 50 & 6,3 & $\begin{array}{l}0 \\
7\end{array}$ & 4 & 2,4 & 75.0 \\
\hline Aerob & 5.6 & 60 & 50 & 18,8 & $\begin{array}{l}2, \\
0\end{array}$ & 4 & 2,4 & 30 \\
\hline $\begin{array}{l}\text { Final } \\
\text { Sedimentation }\end{array}$ & 3 & 0 & 50 & 6,3 & $\begin{array}{l}0 \\
7\end{array}$ & 4 & 6,3 & 30 \\
\hline
\end{tabular}

Table 4 shows the early was TSS $250 \mathrm{mg} / \mathrm{L}$, after processed by Aerobic Anaerobic WWTP constantly become $30 \mathrm{mg} / \mathrm{L}<50 \mathrm{mg} / \mathrm{L}$ (qualify for First-Class river quality standard according to PP 82/2001)

Table 5. Wastewater with TSS $678 \mathrm{mg} / \mathrm{L}$

\begin{tabular}{|c|c|c|c|c|c|c|c|c|}
\hline \multirow[b]{2}{*}{ Name of Tub } & \multirow{2}{*}{$\begin{array}{c}\text { DT } \\
\text { (hour) }\end{array}$} & \multirow{2}{*}{$\begin{array}{l}\text { Efficie } \\
\text { ncy } \\
\quad(\%)\end{array}$} & \multirow{2}{*}{$\begin{array}{l}\text { Debit } \\
\left(\mathrm{m}^{3} /\right. \\
\text { day })\end{array}$} & \multirow{2}{*}{$\begin{array}{c}\text { Effectiv } \\
\mathrm{e} \\
\text { volume } \\
\left(\mathrm{m}^{3}\right)\end{array}$} & \multicolumn{3}{|c|}{ Dimension } & \multirow{2}{*}{$\begin{array}{l}\text { TSS } \\
\text { result } \\
\text { s } \\
(\mathrm{mg} / \\
\mathrm{L})\end{array}$} \\
\hline & & & & & $\begin{array}{l}\mathrm{L}( \\
\mathrm{m})\end{array}$ & $\begin{array}{l}\mathrm{W}( \\
\mathrm{m})\end{array}$ & $\begin{array}{c}\text { Height } \\
+ \text { Air } \\
\text { ( m) }\end{array}$ & \\
\hline $\begin{array}{l}\text { Like } \\
\text { equalization }\end{array}$ & 5.12 & 0 & 50 & 1,0 & 2,2 & 1 & 1,4 & 678 \\
\hline $\begin{array}{l}\text { Initial } \\
\text { sedimentation }\end{array}$ & 3 & 25 & 50 & 10,7 & 3,3 & 4 & 2,4 & 508.5 \\
\hline Anaerob & 9 & 80 & 50 & 6,3 & 0,7 & 4 & 2,4 & 101.7 \\
\hline Aerob & 5.6 & 60 & 50 & 18,8 & 2,0 & 4 & 2,4 & 40.7 \\
\hline $\begin{array}{l}\text { Final } \\
\text { Sedimentation }\end{array}$ & 3 & 0 & 50 & 6,3 & 0,7 & 4 & 6,3 & 40.7 \\
\hline
\end{tabular}

Table 5 shows the early TSS was $678 \mathrm{mg} / \mathrm{L}$, after processed by Aerobic Anaerobic WWTP constantly become 40,7 mg / L <50 mg/L (qualify for First-Class river quality standard according to PP 82/2001) From the above table, the following graph is obtained: 


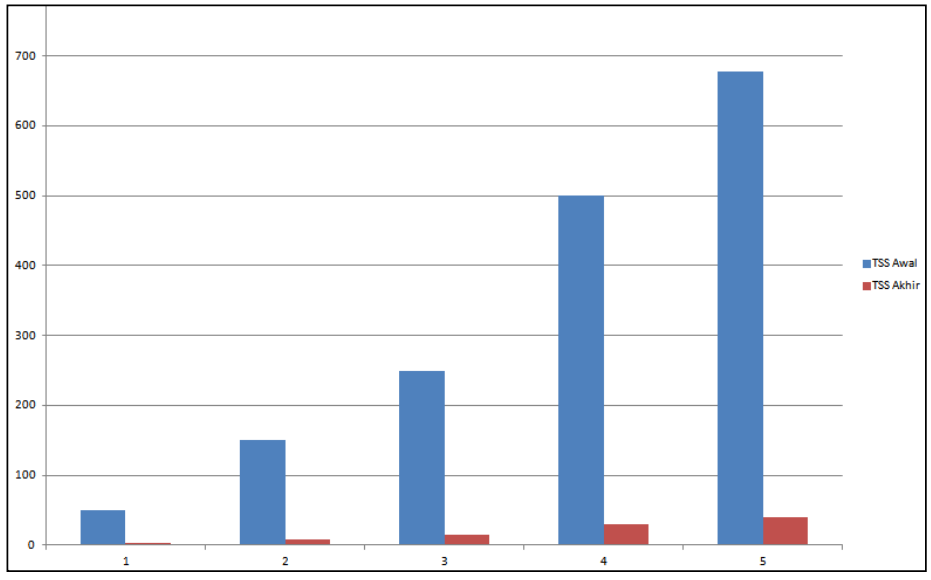

Fig.2 TSS relationship

From the figure 2 above there is significant relation between the early TSS and the final TSS which was so different around $6 \%$

\section{CONCLUSIONS}

In a $50 \mathrm{~m}^{3}$ debit of Textile Wastewater, an Initial TSS value that varies between $50 \mathrm{mg} / \mathrm{L}$ to $678 \mathrm{mg} / \mathrm{L}$ through the anerobic Aerobic WWTP becomes the Final TSS value to be discharged into the Environment to between $3 \mathrm{mg} / \mathrm{L}$ and $40.7 \mathrm{mg} / \mathrm{L}$ meets the standard Class I water quality from the Republic of Indonesia Government Regulation No.82 of 2001.

\section{ACKNOWLEDGMENTS}

On this occasion, we want to thank Ministry of Research and Technology and Director of Semarang State Polytechnic who has given us the opportunity to carry out Community Service activities in the second year.

\section{BIBLIOGRAPHY}

[1] Mochtar Hadiwidodo, Haryono Setyo Huboyo, Indrasarimmawati, 2019, Color Reduction, COD and TSS of Textile Industry Wastewater Using Dielectric Barrier Discharge Technology with Variations in Oxygen Voltage and Flow Rate, Presipitasi Jurnal Vol. 7 No.2 September 2009, ISSN 1907-187X

[2]. Suhartono,E dkk, 2019, Final Report on PPPUD Surabayan Blue Jeans in Efforts to Export Through Environmental Management Cleaner, Green Products and Ecoefficiency in Surabaya, Wonopringgo District, Pekalongan Regency, Polytecnic State of Semarang

[3]. Republic of Indonesia Government Regulation No.82 of 2001, 2001, Regarding Water Quality Management and Water Pollution Prevention.

[4]. SK Menteri LH No.115/2003 tentang Pedoman Penentuan Status Mutu Air.

[5] Ryadi, Slamet, 1984, Pencemaran Air , Penerbit Karya Anda Surabaya

[6]. Suhartono,E and Basuki,SB, 2015, Teknik Drainase Dan Pengelolaan Air Limbah Wilayah Perkotaan Pesisir,Badan Penerbit Polines, ISBN : 978-602-1673-09-6 